\title{
Tangence
}

\section{Enseigner la littérature africaine de langue française dans les universités américaines. Notes à partir d'une expérience en cours}

\section{Amadou Koné}

Numéro 49, décembre 1995

Les littératures francophones de l'Afrique et des Antilles

URI : https://id.erudit.org/iderudit/025875ar

DOI : https://doi.org/10.7202/025875ar

Aller au sommaire du numéro

Éditeur(s)

Tangence

ISSN

0226-9554 (imprimé)

1710-0305 (numérique)

Découvrir la revue

Citer cet article

Koné, A. (1995). Enseigner la littérature africaine de langue française dans les universités américaines. Notes à partir d'une expérience en cours. Tangence, (49), 23-31. https://doi.org/10.7202/025875ar 


\section{Enseigner la littérature africaine de langue française dans les universités américaines. Notes à partir d'une expérience en cours}

\section{Amadou Koné}

Pendant une quinzaine d'années, j'ai enseigné la littérature africaine francophone à l'Université Nationale de Côte d'Ivoire à Abidjan au département des lettres modernes. Je me trouvais alors dans un contexte où la littérature africaine à force de lutter avait fini par "s'émanciper", si je puis dire. En effet, sa présence de la première année de Deug à la Licence et au delà, en faisait une littérature à part entière et qui pouvait s'enseigner de façon progressive et continue, à des niveaux différents, dans une tradition historique, littéraire, et critique.

Il y a un peu plus de trois ans, je me suis retrouvé à La Nouvelle Orleans, en Louisiane, à Tulane University au département de français et d'italien. J'y enseigne la littérature francophone au sud du Sahara. Mon recrutement s'est effectué dans le cadre des efforts déployés par Tulane pour introduire cette littérature dans son curriculum et construire une unité d'enseignement solide dont le professeur Sellin avait posé les bases. Ma nouvelle situation m'a placé d'emblée dans un système américain que je ne connaissais pas. Et j'y ai retrouvé la littérature africaine avec un statut différent de celui que je lui connaissais.

Actuellement, je ne prétends pas encore comprendre parfaitement le système américain. Je ne prétends pas non plus avoir bien compris le statut de la littérature africaine en langue française dans les universités américaines. Je voudrais livrer ici cette expérience encore incomplète, avec les remarques qu'elle suscite en moi et en tenant compte de trois autres expériences qui me paraissent intéressantes à rappeler.

En général et presque dans toutes les universités qui le proposent, l'enseignement de la littérature africaine de langue française ne se fait pas dans un véritable programme, c'est-à-dire une structure homogène et autonome où les cours seraient établis avec une certaine logique de fréquence et d'évolution du 
niveau ${ }^{1}$. En fait un véritable programme autonome me paraît impossible à construire étant donné le statut de ce qu'on appelle ici "la francophonie". C'est en effet ainsi que se nomme notre discipline. Ce qu'on appelle la littérature africaine est une spécialité inclue dans les départements de français, enseignée dans les départements de français. Que la littérature africaine arrive dans les universités américaines par la francophonie s'explique sans doute. Il n'empêche. Cela paraît suspect, et est, dans tous les cas, lourd de conséquences. L'enseignement des littératures africaines entre dans une logique où la francophonie est une manière d'intéresser les étudiants à la langue française par l'attrait d'une culture non occidentale. En fait l'accent est mis sur la langue française non pas tellement sur la littérature africaine. Outre les implications idéologiques qui sont évidentes, un tel statut limite terriblement les possibilités d'expansion de cet enseignement qui finit par n'occuper qu'un aspect du $x^{e}$ français, une place très réduite dans le curriculum.

La littérature africaine d'expression française devient un appendice de la littérature française. Et comme on fonctionne par siècle, elle ne peut occuper qu'une infime parcelle de ce $\mathrm{xx}^{\mathrm{e}}$ siècle français. On a ainsi tendance à croire que le "francophoniste" ne s'occupe que d'un domaine étroit, limité dans le temps et dans l'étendue de son corpus. C'est une erreur énorme. La littérature africaine existe depuis qu'existe l'Africain. Et ce n'est donc pas une littérature du $\mathrm{xx}^{\mathrm{e}}$ siècle uniquement. Est-il nécessaire de dire qu'il s'agit d'une littérature millénaire qui couvre tous les genres occidentaux modernes et même des genres inexistants dans la littérature occidentale comme le "Donso donkili" bambana ou le "didiga " bete, ou le "mvet" fang! Cela tombe sous le bon sens. D'autre part, de la même façon qu'on peut se spécialiser en Rabelais, Hugo, Cervantes, Twain, l'on a suffisamment de

1 Je parle de programme. Je ne parle pas de département de littératures africaines. Or, clans le meilleur des cas, c'est vers de véritables départements de littératures africaines qu'il faudrait aboutir au plus vite dans les universités africaines, et ailleurs plus tard. Car même à Abidjan, la littérature africaine n'est pas complètement autonome dans le Département des lettres modernes. L'autonomie ne serait pas une question simplement idéologique. Mais elle donnerait la liberté et la latitude à cet enseignement de proposer des concepts nouveaux, d'inventer des méthodologies nouvelles et enrichissantes, initiatives que la tutelle de la littérature française et des études françaises étouffent même inconsciemment. 
matière pour se spécialiser sur des auteurs tels que Senghor, Césaire $^{2}$, Mongo Beti, Tchikaya U'Tamsi, etc. Mais c'est la perception étroite de cette littérature qui prédomine. Cette perception a des conséquences néfastes sur la discipline.

D'abord, elle explique en partie sa sous-représentation dans le curriculum. Même dans les universités où il y a un véritable effort pour développer cet enseignement de la littérature francophone d'Afrique, comme à Tulane, nous n'échappons pas à ce problème. À Tulane, nous pouvons nous considérer comme favorisés puisque l'unité francophone compte trois enseignants: Le professeurs Eric Sellin qui a mis les bases de l'unité d'enseignement des littératures francophones et qui s'occupe de la littérature du Maghreb et des Caraibes, le professeur Thomas Klingler qui est linguiste et qui s'occupe du créole louisianais et du créole antillais, et moi-même qui m'occupe de la littérature de l'Afrique subsaharienne. Il faut avouer qu'en considérant le nombre des enseignants qui s'en occupent dans le département, la francophonie est bien représentée. Mais le problème se situe au niveau de la répartition des cours. Étant donné le curriculum et aussi le nombre des séminaires qui peuvent être proposés aux étudiants "gradués" (étudiants de troisième cycle), il arrive très souvent qu'on n'offre qu'un seul séminaire en francophonie par semestre. On ne peut pas faire autrement car on offrirait plus de littérature francophone au détriment de la littérature française qui est, à juste titre, la matière la plus importante dans un département de français. D'autre part, ce séminaire est très souvent un survey (cours d'initiation) qui s'adresse autant aux étudiants de troisième cycle qu'aux étudiants les plus avancés de cycle inférieur (Seniors). Évidemment, un tel séminaire échappe difficilement aux généralités. On est en fait conduit à donner quelques rudiments de la littérature francophone et non à offrir un cours de niveau élevé.

Cette perception étroite explique aussi une croyance qui me paraît dangereuse. C'est cette tendance à croire que l'enseignement de la littérature francophone est une espèce de bricolage auquel peut se livrer n'importe qui. Mon expérience aux ÉtatsUnis me fait penser qu'il suffit qu'un professeur, par ailleurs spé-

2 On verra avec l'article de Daniel Racine les difficultés que selui-ci a rencontrées pour couvrir l'ouvre de Cesaire en un semestre. Ce type d'entreprise ne peut mener qu'à un survol de cette ouvre et non à un véritable travail approfondi. Et cela à cause du manque de temps. 
26

cialiste d'un autre domaine, ait lu par ci et par là quelques romans africains, quelques articles sur l'orientalisme et le postcolonialisme pour s'ériger en spécialiste de littérature francophone. Autant cet intérêt pour la littérature africaine est flatteur autant l'amateurisme a de quoi inquiéter. Car autant je ne peux prétendre à être un spécialiste de $\mathrm{xIX}^{\mathrm{e}}$ ou $\mathrm{xx}^{\mathrm{e}}$ français, bien que j'en sache un bout sur cette littérature, à cause de ma formation dans le système français, autant l'on doit reconnaître la vanité de s'improviser spécialiste "francophoniste".

Car, à examiner les choses avec lucidité, l'enseignement de la littérature africaine dans le contexte américain ${ }^{3}$ pose des problèmes autrement complexes. Ces problèmes sont dus à la conception du curriculum dans les universités - j'en ai déjà parlé mais j'illustrerai mes propos bientôt par des exemples -, à la nature de la discipline, au niveau de la connaissance que les étudiants ont de l'Afrique, de son histoire, de sa culture ${ }^{4}$. Les vrais spécialistes sont conscients de ces problèmes.

Tous les témoignages que j'ai pu lire concernant l'enseignement de la littérature francophone aux États-Unis soulignent les nombreuses précautions qu'il faut initialement prendre pour tenter d'atteindre son objectif: c'est-à-dire faire comprendre cette littérature à un public qui n'est pas familier avec l'Afrique. Car, il est essentiel de comprendre que la lecture d'une littérature est un processus intertextuel sur différents niveaux. C'est la mise en rapport de ce qu'on lit avec l'histoire, la culture, la littérature, le genre littéraire, la tradition littéraire d'un pays ou d'une région. Or dans le cadre de l'enseignement francophone en Amérique, le public, les étudiants, ont une connaissance presque nulle de l'Afrique. Comment faire pour "bien" enseigner un texte africain : un roman, une pièce de théâtre, un poème, en deux semaines dans ces conditions?

Le témoignage d'Eric Sellin qui a enseigné dans ces conditions le roman de Chinua Achebe, Things Fall Apart, est révélateur ${ }^{5}$.

3 Il en serait sans doute de même dans tout contexte étranger où la connaissance de l'histoire, de la culture africaine est assez réduite. Disons dans tout pays différent des anciennes puissances coloniales.

4 Je parle de l'Afrique, mais il en serait de même pour le Vietnam, le Cambodge, le Liban, etc.

5 Eric Sellin. "Teaching Things Fall Apart in the Humanities Core Course ", dans Approaches to Teaching Achebe's Things Fall Apart, Bernth Lindsfors (dir.), 
Sur les six séances disponibles pour l'étude du roman, Eric Sellin en a effectivement consacré deux au roman d'Achebe. Les deux premières séances ont été consacrées à l'histoire de l'Afrique, de la colonisation de l'Afrique, du XIV ${ }^{\mathrm{e}}$ siècle à la Conférence de Berlin, à la pénétration économique, politique et militaire de l'Afrique. La troisième séance a été consacrée à la lecture de quelques chapitres d'un livre susceptible d'aider à la compréhension de Things Fall Apart. La quatrième séance est consacrée à la décolonisation, à la psychologie du colonisé, etc. Ce sont en fait les deux dernières séances qui effectivement ont été effectivement consacrées à l'étude du roman d'Achebe. On pourrait penser qu'il y a un déséquilibre entre l'étude du contexte et l'étude du roman. Mais qui connait le texte comprend qu'Eric Sellin a parfaitement eu raison de procéder comme il l'a fait. Comment comprendre Things Fall Apart sans connaitre un minimum de l'histoire de la colonisation de l'Afrique, du rôle des missionnaires dans "l'œuvre de civilisation occidentale"? Comment parler de tout cela sans parler de la lutte anticoloniale, des textes de Fanon, du Marxisme? Comment ne pas faire référence à d'autres textes d'Achebe, d'autres textes littéraires africains où ce thème fondamental de la littérature africaine francophone ou anglophone se trouve à profusion? En vérité, en six séances, il paraît impossible d'étudier convenablement ce roman dans un contexte où la littérature africaine n'est pas déjà étudiée à d'autres niveaux, dans un contexte où l'on ne connaît pas la culture de base. En fait, la démarche d'Eric Sellin est celle de tous ceux qui connaissent la littérature africaine et veulent l'enseigner dans les universités américaines. C'est aussi ce qui ressortit du second témoignage que je voudrais rapporter ici.

Il s'agit du témoignage de Daniel Racine sur un cas précis d'un cours qu'il a eu à concevoir et à offrir. Il explique en effet dans son article, "L'enseignement de la littérature africaine d'expression française au niveau universitaire: quelques suggestions ", les problèmes qu'ils a rencontrés quand on lui a demandé d'enseigner un cours de "littérature noire" d'expression française à Yale.

New York, The Modem Language Association of America, 1991, p. 118-122. On me dira qu'il s'agit ici d'un roman africain anglophone. Mais à ce niveau, il n'est pas important que le texte africain en question soit anglophone. Les obstacles soulignés sont les mêmes que ceux rencontrés dans l'enseignement des textes francophones. 
J'avoue que c'est un tour de force que de présenter pour un cours de quelques semaines un programme qui soit exhaustif et qui ne soit pas trop chargé pour ne pas décourager les étudiants, et susceptible de les séduire. Il fallait tenir compte de l'aspect historique de cette littérature sans oublier les circonstances de sa naissance, de l'enthousiasme de ses pionniers, de certains facteurs qui ont pu les motiver, et de ses aspirations. J'en suis venu à la description suivante: Genèse, dynamique, dialectique, influence et tendance de la littérature noire d'expression française par l'étude de quelques ouvres représentatives appartenant à la poésie, au roman, au théâtre, à l'essai. ${ }^{6}$

Un peu plus loin Daniel Racine montre également la difficulté qu'il a rencontrée à choisir les textes. Ce cours est le type de survey que l'on donne le plus souvent dans les enseignements francophones. Comme on le voit, en un semestre, il faut tenter de donner aux étudiants une connaissance suffisante sur la littérature de tout un continent. Imaginons un peu qu'on demande à un spécialiste de littérature française ou anglaise de faire la même chose avec la littérature française ou anglaise dans un contexte où l'histoire et la culture françaises ou anglaises seraient pratiquement inconnues. Dans ce sens, ce serait une chose choquante, n'est-ce pas? Je crois qu'une telle chose ne serait pas demandée par les universités si l'on n'avait pas la perception étroite de la littérature africaine, perception dont j'ai déjà parlé. Dans tous les cas, donner un tel cours est un tour de force qui malgré la compétence du professeur, son habileté à choisir, à synthétiser, ne peut aboutir qu'à une présentation partielle et superficielle de la littérature africaine.

Christopher Miller à qui ont doit déjà l'impressionnante étude, Theories of Africains, Francophone Literature and Anthropologie in Africa (1990), a, dans un article intitulé "Literary Studies and African Literature: The Challenge of Intercultural Literacy ${ }^{7}$, analysé la place des études littéraires africaines dans les

6 Daniel Racine. "L'enseignement de la littérature africaine d'expression française au niveau universitaire: quelques suggestions", The Teaching of African Literature, Thomas Hale et Richard Priebe (dir.), Washington, D.C., Three Continents Press and The African Literature Association, 1989, p. 13.

7 Christopher L. Miller, "Literary Studies and African Literature: The Challenge of Intercultural Literacy", dans Africa and the Disciplines, Robert H, 13ates, V.Y. Mudimbe et Jean O'Barr (dir.), Chicago et Londres, The University of Chicago Press, 1992, p. 213-231. 
universités américaines. L'article de Miller est à la fois historique et analytique. L'évocation de l'histoire de la discipline aux ÉtatsUnis montre les résistances qui se sont élevées contre l'introduction même de la littérature africaine dans l'université américaine et décrit la position conservatrice selon laquelle le mouvement vers la diversité était déjà allé trop loin. Miller n'a aucun mal à réfuter de telles idées. Mais l'article de Miller me paraît autrement fondamental à cause sa problématique centrale:

What does the study of African literature bring to the field of literary studies? Does it provide anything more than a vast new supply of raw materials (texts) to which Western methodologies can now be applied? Or does African literature pose more profund challenges? ${ }^{8}$

[Qu'est-ce que l'étude de la littérature africaine apporte au champ des études littéraires? Apporte-t-elle quelque chose d'autre de plus qu'une vaste masse de matière première (textes) qui peut être soumise aux méthodologies occidentales? Ou alors la littérature africaine pose-t-elle des problèmes plus profonds?]

Miller, à mon avis répond de façon tout à fait satisfaisante en montrant qu'évidemment, la nature même de la littérature africaine entraîne un nombre important de problèmes qui amènent le chercheur à réexaminer les concepts de base de la littérature en commençant par le terme de "littérature" lui-même. Et que les méthodologies occidentales ne pouvaient pas s'appliquer de manière mécanique à cette littérature.

Mon expérience d'enseignant de littérature africaine aux États-Unis est semblable à celle de mes deux collègues dont j'ai parlé au départ. D'autre part, mes préoccupations, quand j'ai commencé à enseigner cette littérature, rejoignent parfaitement celles de Christopher Miller.

Le premier séminaire que j'ai eu à donner à Tulane était offert à des étudiants "gradués" (de troisième cycle). C'était déjà une chance pour moi. C'étaient des étudiants capables de lire correctement le français, des étudiants motivés car ils avaient volontairement choisi de suivre ce séminaire. Pendant la première séance, j'ai tenté de faire le point sur ce qu'ils savaient de l'Afrique, de son histoire, de sa culture, de sa littérature. Trois étudiants seulement 
sur douze avaient lu un roman africain. Personne n'avait aucune idée de l'histoire de l'Afrique de l'ouest francophone. Et ce séminaire devait durer un semestre. J'avais la liberté de choisir un sujet à ma convenance et je choisis le sujet suivant: "La littérature africaine de l'oral à l'écrit".

Les raisons d'un tel choix sont simples et à mon avis facilement justifiables. Je crois qu'il faut faire comprendre aux étudiants qui commencent à découvrir la littérature africaine qu'il existe une littérature vaste, complexe, sophistiquée en langue africaine et que cette littérature orale a toujours existé avant que ne naisse la littérature dans les langue des anciennes puissances coloniales. C'est par là qu'il faut commencer. En commençant par là, on aborde, peut-être sommairement il est vrai, les questions que je crois essentielles. En effet, poser le problème de la littérature orale, c'est poser le problème essentiel de la littérarité en d'autres termes. Qu'est-ce que la littérature? L'idée de littérature telle qu'elle a été conceptualisée par la tradition occidentale écrite est-elle universelle? En vérité, les débats qui se sont déroulés autour de l'expression "littérature orale", autour de l'acceptation du corpus de textes oraux comme textes littéraires, ces débats ne me semblent nullement dépassés et n'ont certes pas épuisé la question. Viennent ensuite les questions corollaires qui ne sont pas moins importantes du reste. Passage de l'oral à l'écrit en littérature, passage d'une langue à une autre et spécifiquement passage de la langue africaine à la langue de l'ex-colonisateur dans un contexte de bilinguisme sournoisement considéré comme voulu par les Africains, considérations idéologiques, esthétiques. Et finalement la question critique. On le voit, la question du statut de la littérature africaine nous ramène à l'essence de la littérature. Christopher Miller a parfaitement raison quand il pose dans son article déjà cité la thèse selon laquelle

[...] the study of Africa demands nothing less than a reconsideration of all the terms of literary analysis, starting with the word "literature" itself, and [...] such a reconsideration is the best thing that can happen to the field. ${ }^{9}$

[L'étude de l'Afrique ne demande pas moins qu'une reconsidération de tous les termes de l'analyse littéraire, à commencer par le mot "littérature" lui-même, et [...] une telle reconsidération est la meilleure chose qui puisse arriver au champ littéraire]

9 Ibid., p. 217. 
Et c'est en effet ce qui est apparu dans ce séminaire qui, sans prétention, a conduit les étudiants à revenir sur des concepts qui leur semblaient canonisés, figés de façon définitive. Et c'est aussi l'aspect positif dans l'enseignement ici au États-Unis. Les étudiants sont souples et capables de réviser les concepts. Ainsi à défaut d'avoir résolu les problèmes, nous avons pu discuter de façon profitable, me semble-t-il, sur ces questions complexes. Et je crois que l'étude la littérature africaine a poussé les étudiants à acquérir une certaine richesse ne serait qu'au niveau des concepts littéraires.

Que la place de cette littérature dans le curriculum soit limitée est un fait. Que les professeurs de littérature africaine apparaissent aussi comme des marginaux et des non spécialistes, cela est souvent vrai. Mais le "francophoniste" doit s'estimer heureux car son domaine se développe très rapidement. De nombreuses universités américaines le proposent à leurs étudiants. Cela semble être l'aboutissement de la longue lutte des francophonistes qu'a décrite Christopher Miller. Il faut espérer que la qualité de cet enseignement va s'améliorer progressivement, s'élever du stade des introductions générales à celui d'un enseignement de spécialistes et de haut niveau. Pour ce qui concerne les ressources humaines, il n'y a pas d'inquiétude à se faire. Il y a ici aux États-Unis, dans le domaine de la littérature africaine francophone, des professeurs brillants, compétents, érudits, et qui aiment leur domaine. J'ai rencontré aussi des étudiants brillants et motivés qui s'intéressent à la discipline, s'y spécialisent. Nul doute qu'ils sauront triompher des obstacles. Et en considérant certains travaux déjà faits par les Américains, je suis persuadé qu'effectivement le renouvellement des méthodologies critiques et des théories littéraires se fera ici à travers la littérature africaine. 